\section{Keine Körperverletzung durch einmalige Röntgenuntersuchung}

\section{Einführung}

Es ist wissenschaftlich anerkannt, dass ionisierende Strahlen, die auf den menschlichen Körper treffen, dort Zellen schädigen oder abtöten und Erbanlagen verändern können. Das Vorliegen einer pathologischen Gesundheitsbeeinträchtigung durch Röntgenuntersuchungen ist jedoch angesichts der geringen Strahlendosen, die bei ordnungsgemäß durchgeführten diagnostischen Röntgenuntersuchungen auftreten, nur schwer und in der Regel nicht nachweisbar. Insofern stellt sich sowohl für den Radiologen als auch für das medizinische Fachpersonal wie MTRA und MFA in der täglichen Praxis die Frage, ob und unter welchen Voraussetzungen eine zivilrechtliche Haftung für eine fehlerhafte Röntgenuntersuchung bestehen kann.

In einem früheren Beitrag waren Haftungsfragen bei diagnostischen Röntgenuntersuchungen bereits ein ausführliches Thema (vgl. Wigge, Loose, RöFo 2/2016, S. 218 ff. und 3/ 2016, S. 312 ff.). Schwerpunkte der Darstellung waren das Aufklärungserfordernis im Hinblick auf eine wirksame Einwilligung in die Untersuchung, das Gefährdungspotenzial von Röntgenstrahlen bei diagnostischen Anwendungen, die Rechtsprechung zur Aufklärungspflicht bei Röntgenuntersuchungen, die Voraussetzungen der Röntgenverordnung (RöV) zur Aufklärung und die Delegationsfähigkeit der Aufklärung. Anlässlich einer kürzlich ergangenen Entscheidung des Amtsgerichts (AG) Paderborn vom 15.03.2019 (Az.: 58a C 155/17) zu streitigen Ansprüchen infolge einer Röntgenuntersuchung und der zwischenzeitlichen Novellierung des Strahlenschutzrechts, insbesondere durch das Strahlenschutzgesetz (StrlSchG) und die neue Strahlenschutzverordnung (StrlSchV), befasst sich dieser Beitrag erneut mit der Thematik der Körperverletzung durch Röntgenuntersuchungen und damit zusammenhängender Fragen wie der Aufklärungspflicht.

\section{Urteil des AG Paderborn}

In dem zugrunde liegenden Sachverhalt wurde der Patient und spätere Kläger, selbst Chirurg und Unfallchirurg, an der HWS (HWK 5/6) operiert. Ärzte der beklagten radiologischen Gemeinschaftspraxis hatten vor dieser Operation mehrere MRTAufnahmen angefertigt. Nach der Operation fertigte die MTA bei der Kontrolle in der Gemeinschaftspraxis eine Dens-ZielAufnahme bei dem Patienten. Der Patient behauptete, die von der Gemeinschaftspraxis durchgeführte Röntgenuntersuchung sei unnötig und nicht indiziert gewesen. Es hätte nur die HWS in 2 Ebenen geröntgt werden sollen. Die gefertigte Dens-ZielAufnahme sei nicht nachvollziehbar, da bei dieser Aufnahme der betroffene HWK 5/6 nicht zu sehen sei. Nur weil der Patient am Hals operiert worden sei, habe nicht darauf geschlossen werden können, dass möglicherweise Strukturen am Hals verletzt worden seien. Durch diese unnötige Strahlenexposition würden sich Folgeschäden entwickeln. Der Patient beantragte u. a., die Gemeinschaftspraxis zu verurteilen, an den Patienten ein in das Ermessen des Gerichts gestelltes Schmerzensgeld in Höhe von mindestens 1750,00 Euro zu zahlen und festzustellen, dass die Gemeinschaftspraxis alle weiteren zukünftigen materiellen sowie immateriellen Schäden infolge der Untersuchung zu ersetzen habe.

Das Gericht wies die Klage ab. Der Patient habe gegen die Gemeinschaftspraxis weder einen Anspruch aus einer Pflichtverletzung des Behandlungsvertrags gemäß §§630a, 280 Abs. 1 BGB, noch aus einer unerlaubten Handlung gemäß $\S 823$ Abs. 1, Abs. 2 BGB i. V. m. §229 StGB (fahrlässige Körperverletzung), da kein Behandlungsfehler vorliege. Die rechtfertigende Indikation für die generelle Anwendung der Röntgenstrahlung nach dem damals anwendbaren §23 Abs. 1 S. 1 RöV war zwischen den Parteien unstreitig.

In der konkreten Durchführung der Röntgenuntersuchung, gegen die sich der Patient wendete, sah das Gericht keine Pflichtverletzung begründet. Standardmäßig würden in solchen Fällen konventionelle Röntgenaufnahmen der HWS in 2 Ebenen (a. p.-Projektion und seitlich) durchgeführt. Die hier gefertigte Dens-Spezial-(Ziel-)Aufnahme sei eine Zusatzaufnahme zur a.p.-

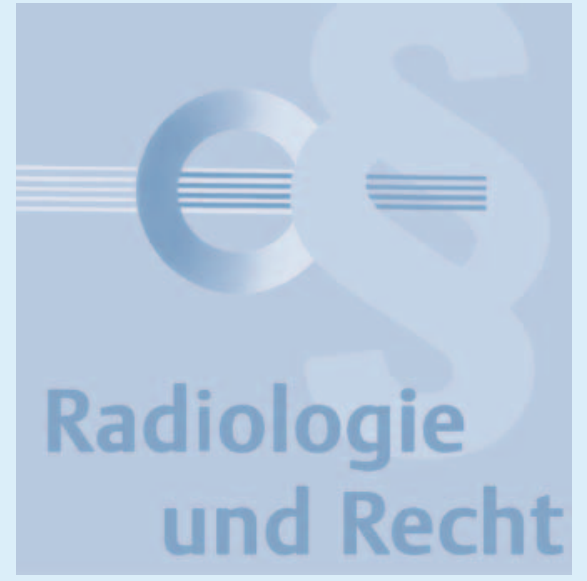

Projektion, die zur überlagerungsfreien Darstellung des Dens axis angefertigt werde. Im vorliegenden Fall sei zwar eine modifizierte Aufnahmereihenfolge gewählt worden, indem mit der Zusatzaufnahme begonnen wurde. Diese wäre nicht zwingend erforderlich gewesen, da eine postoperative Kontrolle ohne die Anfertigung der Dens-Zielaufnahme möglich gewesen wäre. Allerdings ließ sich sachverständigenseits nicht feststellen, dass zwingend eine andere als die hier angewendete Reihenfolge vorgeschrieben sei. Diese zusätzliche Aufnahme führe nämlich zu einer sehr hohen Qualität der Abbildung der gesamten HWS und verbessere die Qualität der Aufnahme in jedem Fall, zumal der Sachverständige nachvollziehbar ausgeführt habe, dass möglicherweise die umgekehrte Fragestellung zu Problemen führen könnte, wenn nämlich die Zusatzaufnahme unterlassen worden wäre. Dann hätten die Aufnahmen in jedem Fall eine insgesamt schlechtere Qualität aufgewiesen. Es hätten dann im Ansatz möglicherweise bestehende andere Erkrankungen nicht festgestellt werden können.

Bemerkenswert ist der Hinweis des Gerichts, dass zudem zu berücksichtigen sei, dass durch die Zusatzaufnahme eine äußerst geringe Strahlendosis vom Patienten aufgenommen worden ist. Die Dens-Zielaufnahme entspreche dem Abbildungsumfang von ca. $25 \%$ einer Röntgenaufnahme der HWS. Besonders hervorzuheben ist sodann die folgende Feststellung des Gerichts:

„Auch absolut gesehen handelt es sich hierbei um eine zu vernachlässigende Strahlendosis. Diese zusätzliche Aufnahme führt zu einer Be- 
lastung von ca. 0,05 mSV. Dies führt in Relation zu der jährlichen Strahlenbelastung in Deutschland aus natürlichen und zivilisatorischen Faktoren von ca. 4mSV pro Jahr zu einer derart geringen Zusatzbelastung, dass diese den Mehrwert der erhöhten Qualität der Röntgenaufnahmen auf jeden Fall rechtfertigt. “

Das Amtsgericht Paderborn folgt damit dem bereits in einem Urteil aus 1997 formulierten Grundgedanken des Bundesgerichtshofs (BGH). Dort wertete der BGH wegen der Vielzahl von medizinisch nicht erforderlichen Röntgenuntersuchungen die Tat eines Orthopäden in dem besonderen Fall zwar als vorsätzliche Körperverletzung i. S. v. §223 StGB, stellte aber abgesehen davon fest, dass

„die einmalige, kurzzeitige oder nur gelegentlich wiederholte ordnungsgemäße Anwendung von Röntgenstrahlen“

in der Regel noch nicht als Körperverletzung zu beurteilen ist (BGH, Urt. v. 03.12.1997-2 StR 397/97, MedR 1998, 326 ff.).

\section{Gefährdungspotenziale von Röntgenstrahlen bei diag- nostischen Anwendungen}

Festzuhalten ist daher, dass zwar die abstrakte Schädigungsmöglichkeit durch ionisierende Strahlen, die auf den menschlichen Körper treffen, feststeht. Allerdings ist das Vorliegen einer pathologischen Gesundheitsbeeinträchtigung durch Röntgenuntersuchungen angesichts der geringen Strahlendosen, die bei ordnungsgemäß durchgeführten diagnostischen Röntgenuntersuchungen auftreten, nur schwer und in der Regel nicht nachweisbar. Voraussetzung für eine Körperverletzung wäre nämlich nicht lediglich das Vorliegen einer abstrakten Schädigungseignung der durchgeführten Röntgenuntersuchung, sondern ein nachweisbarer Strahlenschaden im medizinischen Sinne. Schließlich ist der Kausalzusammenhang zwischen etwaigen Veränderungen und der Strahleneinwirkung nachzuweisen. Dazu müssten die molekularen Zellzustände unmittelbar vor und unmittelbar nach der Untersuchung bekannt und sonstige andere relevante, also poten- ziell zelländernde Strahleneinwirkungen, ausgeschlossen sein.

Entscheidend für die Gesundheitsgefährdung im Bereich niedriger Strahlendosen sind in erster Linie „stochastische“ Strahlenschäden. Diese beruhen auf nicht reparierten Schäden der betroffenen Zellen und können zu neoplastischen Veränderungen, insbesondere strahlenbedingten Krebskrankheiten führen. Bis sich eine Krebserkrankung klinisch manifestiert, vergehen nach der Strahlenexposition allerdings im Durchschnitt 15 bis 25 Jahre. Außerdem ist ein durch Strahlung verursachtes Karzinom klinisch nicht von anderen zu unterscheiden. Von einer Strahlenbedingtheit einer Erkrankung, also einer Wahrscheinlichkeit für eine Strahleninduktion von mindestens $50 \%$, kann bei röntgendiagnostischen Untersuchungen auch bei mehrfacher Anwendung nicht ausgegangen werden (vgl. Jung, Wigge, MedR 1998, 329, 330).

Die körperlichen Veränderungen, die durch die Strahlendosen von diagnostischen Röntgenuntersuchungen verursacht werden können, liegen in der Regel im nicht nachweisbaren Bereich und haben - bezogen auf Befinden und Funktion des Körpers der Betroffenen - grundsätzlich keine pathologisch relevante Bedeutung. Von kleinen Strahlendosen gehen anerkanntermaßen keine nachweisbaren Wirkungen aus; ab etwa 0,2Sv sind Chromosomenveränderungen nachweisbar, ab etwa $0,5 \mathrm{~Sv}$ sind geringfügige Blutbildveränderungen beobachtbar, die allerdings nach Stunden bis wenigen Tagen durch Reparaturvorgänge des Körpers wieder verschwunden sind (Wigge, Loose, RöFo 2/2016, S. 221; vgl. Riemer, Einflüsse epidemiologischer Forschung auf das Strahlenschutzrecht, Diss. 2005, S. 42 m. w. N.). Ein für eine Körperverletzung erforderlicher, nicht ganz unerheblicher Gesundheitsschaden wird hierdurch nicht begründet. Darüber hinaus ist bei geringen Strahlendosen keine eigenständige Bewertung gegenüber der in jedem Fall bestehenden vergleichbaren oder regelmäßig wesentlich intensiveren Umgebungsstrahlung möglich, wie auch aus dem Urteil des AG Paderborn hervorgeht.

\section{Folgen für die ärztliche Aufklärung}

$\S 630$ d Abs. 1 S. 1 regelt die grundsätzliche vertragliche Pflicht des Behandelnden, die Einwilligung des Patienten „vor Durchführung einer medizinischen Maßnahme“ einzuholen. Die Wirksamkeit der Einwilligung setzt voraus, dass der Einwilligende zuvor aufgeklärt worden ist ( $§ 630 \mathrm{~d}$ Abs. 2 BGB). Prinzipiell sind nach $\S 630$ e Abs. 1 BGB die Patienten über „sämtliche für die Einwilligung wesentlichen Umstände“ aufzuklären.

Wie das AG Paderborn deutlich gemacht hat, muss eine Abwägung stattfinden zwischen der Strahlendosis und dem Nutzen für den Patienten. Der eingangs genannte Beitrag in der RöFo führt hierzu bereits aus (Wigge, Loose, RöFo 2/2016, S. 220):

„Für die Aufklärung besteht daher nur dann eine Notwendigkeit, wenn durch die ärztliche Maßnahme ein Körper- oder Gesundheitsschaden des Patienten überhaupt kausal begründet werden kann. Dies dürfte sich im Bereich der ionisierenden Strahlen prinzipiell nur mit der Hilfe von Sachverständigen ermitteln lassen, da nach wohl einhelliger Auffassung in der Naturwissenschaft die Frage grundsätzlich nicht mit der Nennung eines bestimmten Wertes beantwortet werden kann. Dosisgrenzwerte, wie sie für beruflich strahlenexponierte Personen existieren, gibt es für Patienten nämlich nicht, da für sie die Strahlenanwendung nicht nur ein Risiko, sondern auch einen medizinischen Nutzen birgt (Riemer, Einflüsse epidemiologischer Forschung auf das Strahlenschutzrecht, Dissertation 2005, S. 75; Jung, Wigge, Urteilsanmerkung, MedR 1998, 329, 330). Vielmehr hängt der quantitative Effekt einer bestimmten Strahleneinwirkung von einer ganzen Reihe modifizierender Faktoren ab (vgl. Reinhardt, Der strafrechtliche Schutz vor den Gefahren der Kernenergie und den schädlichen Wirkungen ionisierender Strahlen, 1989, S. 29, 30).“

Das AG Paderborn hat diese Einschätzung über das Erfordernis einer Abwägung bestätigt. Aus der Feststellung, dass die zugrunde liegende einzelne Röntgenuntersuchung zu einer derart geringen Zusatzbelastung geführt hat, dass diese den Mehrwert der erhöhten Qualität der Aufnahmen auf jeden Fall rechtfertigt und hierdurch 
kein Körper- oder Gesundheitsschaden begründet werden konnte, folgt, dass im Grundsatz keine Aufklärung über eine solche Untersuchung erforderlich ist.

\section{Vorgaben nach §83 StrlSchG und $\S 119$ StrlSchV}

Nichts anderes ergibt sich aus den einschlägigen spezialgesetzlichen Rechtsgrundlagen. Das AG Paderborn legt für die rechtfertigende Indikation zur Anwendung der Röntgenstrahlung den für diesen Sachverhalt noch geltenden §23 Abs. 1 S. 1 RöV zugrunde. Durch die Verordnung zur weiteren Modernisierung des Strahlenschutzrechts vom 29.11.2018 sind die alte Strahlenschutzverordnung und die RöV zum 31.12.2018 außer Kraft getreten (vgl. Wigge, Kirsch, RöFo 4/2019, S. 382 ff.). Stattdessen gelten jetzt insbesondere $\$ 83$ StrISchG und $\S \S 119 \mathrm{f}$. StrlSchV.

Die Gegenüberstellung der Vorschriften zeigt, dass die einschlägigen Normen der RöV, insbesondere $\S \S 23$ und 25 RöV, im Wesentlichen auf $\S 83$ StrlSchG und $\S \S 119 \mathrm{f}$. StrlSchV aufgeteilt und dabei stellenweise spezifiziert wurden (vgl. BT-Drs. 18/11241, $333 \mathrm{f}$. und BR-Drs.423/18, 420 f.). Die Grundsätze über die rechtfertigende Indikation zur Anwendung von Röntgenstrahlung wurden demnach durch die Rechtsnovellierung hinsichtlich der inhaltlichen Anforderungen nicht verändert. Entscheidend ist eine Abwägung mit anschließender Feststellung, dass der gesundheitliche Nutzen der einzelnen Anwendung gegenüber dem Strahlenrisiko überwiegt ( $§ 83$ Abs. 3 Satz 2 StrlSchG).

Diese Voraussetzungen entsprechen den bisherigen Vorgaben der Rechtsprechung für die Risikoaufklärung, wonach „der $\mathrm{Pa}$ tient über den Verlauf des Eingriffes, seine Erfolgsaussichten, seine Risiken und mögliche echte Behandlungsalternativen, wobei auch ein Zuwarten oder Verzicht auf eine Operation eine Alternative darstellen kann“, aufzuklären ist (vgl. BGH NJW 1984, 1784). Demnach können Röntgenuntersuchungen, bei denen der Arzt über die erforderlichen $\mathrm{Er}$ kenntnisse über den Patienten verfügt und bei denen keine besonderen Abwägungserfordernisse bestehen, grundsätzlich auch ohne Aufklärung durchgeführt werden können.

Besonderheiten sind jedoch dann zu beachten, wenn die speziellen Voraussetzungen der einschlägigen Vorschriften, insbesondere der StrlSchV, erfüllt sind. Beispielsweise bei Vorliegen möglicher Kontraindikationen, vornehmlich bei bereits früher durchgeführten Untersuchungen, §119 Abs. 2 StrlSchV, und (potenzieller) Schwangerschaft, $§ 120$ Abs. 1 und 2 StrlSchV, besteht eine besondere Befragungspflicht und daraus möglicherweise die gesteigerte Beachtung des Minimierungsgebotes der Strahlenbelastung.

\section{Fazit}

Zusammenfassend ist festzuhalten, dass einmalige Röntgenuntersuchungen grundsätzlich nicht geeignet sind, eine Körperverletzung zu verursachen, sodass der $\mathrm{Pa}$ tient vor der Untersuchung auch nicht in jedem Einzelfall gemäß §630 d Abs. 1 S. 1 BGB aufzuklären ist. Prinzipiell gilt diese Einschätzung auch für den Fall, dass die Untersuchung im Ergebnis nicht medizinisch indiziert war. Unabhängig davon sind die in Einzelfällen bestehenden Befragungs- und Aufklärungspflichten zum Schutz von besonderen Personengruppen nach $\S \S 119$ Abs. 3, 120 StrlSchV, z. B. bei häufigen Voruntersuchungen oder etwaigen Schwangeren, durch den Radiologen zu beachten.

Prof. Dr. Peter Wigge

Rechtsanwalt

Fachanwalt für Medizinrecht

Tilmann Kirsch

Rechtsanwalt

Rechtsanwälte Wigge

Großer Burstah 42

20457 Hamburg

Telefon: (040) 3398 705-90

Telefax: (040) 3398 705-99

Internet: www.ra-wigge.de

E-Mail: kanzlei@ra-wigge.de 